$\mathbf{T}_{\text {he }} \mathbf{J}_{\text {ournal of }} \mathbf{N}_{\text {onlinear }} \mathbf{S}_{\text {ciences and }}$ Applications

http://www.tjnsa.com

\title{
CONVERGENCE OF IMPLICIT RANDOM ITERATION PROCESS WITH ERRORS FOR A FINITE FAMILY OF ASYMPTOTICALLY QUASI-NONEXPANSIVE RANDOM OPERATORS
}

\author{
GURUCHARAN SINGH SALUJA
}

\begin{abstract}
In this paper, we prove that an implicit random iteration process with errors which is generated by a finite family of asymptotically quasinonexpansive random operators converges strongly to a common random fixed point of the random operators in uniformly convex Banach spaces.
\end{abstract}

\section{INTRODUCTION}

Random nonlinear analysis is an important mathematical discipline which is mainly concerned with the study of random nonlinear operators and their properties and is needed for the study of various classes of random equations. The study of random fixed point theory was initiated by the Prague school of Probabilities in the 1950s [12, 13, 25]. Common random fixed point theorems are stochastic generalization of classical common fixed point theorems. The machinery of random fixed point theory provides a convenient way of modeling many problems arising from economic theory (see e.g. [19]) and references mentioned therein. Random methods have revolutionized the financial markets. The survey article by Bharucha-Reid [9] attracted the attention of several mathematicians and gave wings to the theory. Itoh [15] extended Spacek's and Hans's theorem to multivalued contraction mappings. Now this theory has become the full fledged research area and various ideas associated with random fixed point theory are

Date: Received: May 21, 2009; Revised: November 12, 2011.

${ }^{*}$ Corresponding author

(c) 2011 N.A.G.

2000 Mathematics Subject Classification. 47H09, 47H10, 47J25.

Key words and phrases. Asymptotically quasi nonexpansive random operator, common random fixed point, implicit random iteration scheme with errors, strong convergence, uniformly convex Banach space. 
used to obtain the solution of nonlinear random system (see $[4,5,8,14,21]$ ). Papageorgiou [17, 18], Beg [2,3] studied common random fixed points and random coincidence points of a pair of compatible random operators and proved fixed point theorems for contractive random operators in Polish spaces. Recently, Beg and Shahzad [7], Choudhury [11] and Badshah and Sayyed [1] used different iteration processes to obtain random fixed points. More recently, Beg and Abbas [6] studied common random fixed points of two asymptotically nonexpansive random operators through strong as well as weak convergence of sequence of measurable functions in the setup of uniformly convex Banach spaces. Also they construct different random iterative algorithms for asymptotically quasi-nonexpansive random operators on an arbitrary Banach space and established their convergence to random fixed point of the operators.

In 2001, $\mathrm{Xu}$ and Ori [27] have introduced an implicit iteration process for a finite family of nonexpansive mappings in a Hilbert space $H$. Let $C$ be a nonempty subset of $H$. Let $T_{1}, T_{2}, \ldots, T_{N}$ be self-mappings of $C$ and suppose that $\mathcal{F}=\cap_{i=1}^{N} F\left(T_{i}\right) \neq \emptyset$, the set of common fixed points of $T_{i}, i=1,2, \ldots, N$. An implicit iteration process for a finite family of nonexpansive mappings is defined as follows, with $\left\{t_{n}\right\}$ a real sequence in $(0,1), x_{0} \in C$ :

$$
\begin{aligned}
x_{1} & =t_{1} x_{0}+\left(1-t_{1}\right) T_{1} x_{1}, \\
x_{2} & =t_{2} x_{1}+\left(1-t_{2}\right) T_{2} x_{2}, \\
& \vdots \\
x_{N} & =t_{N} x_{N-1}+\left(1-t_{N}\right) T_{N} x_{N}, \\
x_{N+1} & =t_{N+1} x_{N}+\left(1-t_{N+1}\right) T_{1} x_{N+1},
\end{aligned}
$$

which can be written in the following compact form:

$$
x_{n}=t_{n} x_{n-1}+\left(1-t_{n}\right) T_{n} x_{n}, \quad n \geq 1
$$

where $T_{k}=T_{k \bmod N}$. (Here the $\bmod \mathrm{N}$ function takes values in $\left.\mathcal{N}\right)$. And they proved the weak convergence of the process (1.1).

In 2003, Sun [23] extend the process ( 1.1) to a process for a finite family of asymptotically quasi-nonexpansive mappings, with $\left\{\alpha_{n}\right\}$ a real sequence in $(0,1)$ and an initial point $x_{0} \in C$, which is defined as follows: 


$$
\begin{aligned}
x_{1} & =\alpha_{1} x_{0}+\left(1-\alpha_{1}\right) T_{1} x_{1}, \\
& \vdots \\
x_{N} & =\alpha_{N} x_{N-1}+\left(1-\alpha_{N}\right) T_{N} x_{N}, \\
x_{N+1} & =\alpha_{N+1} x_{N}+\left(1-\alpha_{N+1}\right) T_{1}^{2} x_{N+1}, \\
& \vdots \\
x_{2 N} & =\alpha_{2 N} x_{2 N-1}+\left(1-\alpha_{2 N}\right) T_{N}^{2} x_{2 N}, \\
x_{2 N+1} & =\alpha_{2 N+1} x_{2 N}+\left(1-\alpha_{2 N+1}\right) T_{1}^{3} x_{2 N+1},
\end{aligned}
$$

which can be written in the following compact form:

$$
x_{n}=\alpha_{n} x_{n-1}+\left(1-\alpha_{n}\right) T_{i}^{k} x_{n}, \quad n \geq 1
$$

where $n=(k-1) N+i, i \in \mathcal{N}$.

Sun [23] proved the strong convergence of the process (1.2) to a common fixed point, requiring only one member $T$ in the family $\left\{T_{i}: i \in \mathcal{N}\right\}$ to be semicompact. The result of Sun [23] generalized and extended the corresponding main results of Wittmann [26] and $\mathrm{Xu}$ and Ori [27].

The purpose of this paper is to introduce and study an implicit random iteration process with errors which converges strongly to a common random fixed point of a finite family of asymptotically quasi-nonexpansive random operators in uniformly convex Banach spaces. Our results extend and improve the corresponding results of Beg and Abbas [5] and many others.

\section{Preliminaries}

Let $(\Omega, \Sigma)$ be a measurable space ( $\Sigma$-sigma algebra) and let $F$ be a nonempty subset of a Banach space $X$. We will denote by $C(X)$ the family of all compact subsets of $X$ with Hausdorff metric $H$ induced by the metric of $X$. A mapping $\xi: \Omega \rightarrow X$ is measurable if $\xi^{-1}(U) \in \Sigma$, for each open subset $U$ of $X$. The mapping $T: \Omega \times X \rightarrow X$ is a random map if and only if for each fixed $x \in X$, the mapping $T(., x): \Omega \rightarrow X$ is measurable and it is continuous if for each $\omega \in \Omega$, the mapping $T(\omega,):. X \rightarrow X$ is continuous. A measurable mapping $\xi: \Omega \rightarrow X$ is a random fixed point of a random map $T: \Omega \times X \rightarrow X$ if and only if $T(\omega, \xi(\omega))=$ $\xi(\omega)$, for each $\omega \in \Omega$. We denote the set of random fixed points of a random map $T$ by $R F(T)$. Let $R F\left(T_{i}\right),(i \in \mathcal{N})$ be the set of all random fixed points of $T_{i}$, where $\mathcal{N}=\{1,2, \ldots, N\}$. The set of common random fixed points of $T_{i}(i \in \mathcal{N})$ denoted by $\mathcal{F}$, that is, $\mathcal{F}=\cap_{i=1}^{N} R F\left(T_{i}\right)$.

Let $B\left(x_{0}, r\right)$ denote the spherical ball centered at $x_{0}$ with radius $r$, defined as the set $\left\{x \in X:\left\|x-x_{0}\right\| \leq r\right\}$. 
We denote the $n t h$ iterate $T(\omega, T(\omega, T(\omega, \ldots, T(\omega, x) \ldots))$,$) of T$ by $T^{n}(\omega, x)$. The letter $I$ denotes the random mapping $I: \Omega \times X \rightarrow X$ defined by $I(\omega, x)=x$ and $T^{0}=I$.

Definition 2.1. Let $C$ be a nonempty separable subset of a Banach space $X$ and $T: \Omega \times C \rightarrow C$ be a random map. The map $T$ is said to be the following:

(a) A nonexpansive random operator if for $x, y \in C$, one has

$$
\|T(\omega, x)-T(\omega, y)\| \leq\|x-y\|
$$

for each $\omega \in \Omega$.

(b) Asymptotically nonexpansive random operator if there exists a sequence of measurable mapping $k_{n}: \Omega \rightarrow[1, \infty)$ with $\lim _{n \rightarrow \infty} k_{n}(\omega)=1$, such that for $x, y \in C$, one has

$$
\left\|T^{n}(\omega, x)-T^{n}(\omega, y)\right\| \leq k_{n}(\omega)\|x-y\|
$$

for each $\omega \in \Omega$.

(c) Asymptotically quasi-nonexpansive random operator if for each $\omega \in \Omega$, $G(\omega)=\{x \in C: x=T(\omega, x)\} \neq \phi$ and there exists a sequence of measurable mapping $r_{n}: \Omega \rightarrow[0, \infty)$ with $\lim _{n \rightarrow \infty} r_{n}(\omega)=0$, such that for $x \in C$ and $y \in G(\omega)$, the following inequality holds:

$$
\left\|T^{n}(\omega, x)-y\right\| \leq\left(1+r_{n}(\omega)\right)\|x-y\|
$$

for each $\omega \in \Omega$.

(d) Uniformly L-Lipschitzian random operator if for $x, y \in C$

$$
\left\|T^{n}(\omega, x)-T^{n}(\omega, y)\right\| \leq L\|x-y\|,
$$

for each $\omega \in \Omega$, where $n=1,2, \ldots$ and $L$ is a positive constant.

(e) A semicompact random operator if for a sequence of measurable mappings $\left\{\xi_{n}\right\}$ from $\Omega$ to $C$, with $\lim _{n \rightarrow \infty}\left\|\xi_{n}(\omega)-T\left(\omega, \xi_{n}(\omega)\right)\right\|=0$, for every $\omega \in \Omega$, one has a subsequence $\left\{\xi_{n_{k}}\right\}$ of $\left\{\xi_{n}\right\}$ and a measurable mapping $\xi: \Omega \rightarrow C$ such that $\left\{\xi_{n_{k}}\right\}$ converges pointwisely to $\xi$ as $k \rightarrow \infty$.

Motivated and inspired by $\mathrm{Xu}$ and Ori [27], Sun [23] and some others, we introduce and study an implicit random iteration scheme with errors as follows:

Definition 2.2. Let $\left\{T_{1}, T_{2}, \ldots, T_{N}\right\}$ be a family of $N$-random operators from $\Omega \times C$ to $C$, where $C$ is a nonempty closed convex subset of a separable Banach space $X$. Let $\left\{f_{n}\right\}$ be a bounded sequence of measurable mappings from $\Omega$ to $C$ and let $\xi_{0}: \Omega \rightarrow C$ be a measurable mapping. Define the random iteration process with errors $\left\{\xi_{n}\right\}$ as follows: 


$$
\begin{aligned}
\xi_{1}(\omega) & =\alpha_{1} \xi_{0}(\omega)+\beta_{1} T_{1}\left(\omega, \xi_{1}(\omega)\right)+\gamma_{1} f_{1}(\omega), \\
\xi_{2}(\omega) & =\alpha_{2} \xi_{1}(\omega)+\beta_{2} T_{2}\left(\omega, \xi_{2}(\omega)\right)+\gamma_{2} f_{2}(\omega), \\
& \vdots \\
\xi_{N}(\omega) & =\alpha_{N} \xi_{N-1}(\omega)+\beta_{N} T_{N}\left(\omega, \xi_{N}(\omega)\right)+\gamma_{N} f_{N}(\omega), \\
\xi_{N+1}(\omega) & =\alpha_{N+1} \xi_{N}(\omega)+\beta_{N+1} T_{1}^{2}\left(\omega, \xi_{N+1}(\omega)\right)+\gamma_{N+1} f_{N+1}(\omega), \\
& \vdots \\
\xi_{2 N}(\omega) & =\alpha_{2 N} \xi_{2 N-1}(\omega)+\beta_{2 N} T_{N}^{2}\left(\omega, \xi_{2 N}(\omega)\right)+\gamma_{2 N} f_{2 N}(\omega), \\
\xi_{2 N+1}(\omega) & =\alpha_{2 N+1} \xi_{2 N}(\omega)+\beta_{2 N+1} T_{1}^{3}\left(\omega, \xi_{2 N+1}(\omega)\right)+\gamma_{2 N+1} f_{2 N+1}(\omega),
\end{aligned}
$$

which can be written in the following compact form:

$$
\xi_{n}(\omega)=\alpha_{n} \xi_{n-1}(\omega)+\beta_{n} T_{i}^{k}\left(\omega, \xi_{n}(\omega)\right)+\gamma_{n} f_{n}(\omega),
$$

where $n=(k-1) N+i, i \in \mathcal{N}$, and each $\left\{f_{n}(\omega)\right\}$ is bounded sequence in $C,\left\{\alpha_{n}\right\}$, $\left\{\beta_{n}\right\},\left\{\gamma_{n}\right\}$ be three appropriate real sequences in $[0,1]$ such that $\alpha_{n}+\beta_{n}+\gamma_{n}=1$ for $n=1,2, \ldots$ Process $(2.5)$ is called the implicit random iteration process with errors for a finite family of random operators $T_{i}(i=1,2, \ldots, N)$.

Recall that a mapping $T: C \rightarrow C$ where $C$ is a subset of $X$ with $F(T) \neq \emptyset$ is said to satisfy condition $(A)[22]$ if there exists a nondecreasing function $f:[0, \infty) \rightarrow[0, \infty)$ with $f(0)=0, f(r)>0$ for all $r \in(0, \infty)$ such that $\|x-T x\| \geq f(d(x, F(T)))$ for all $x \in C$ where $d(x, F(T))=\inf \{\|x-p\|: p \in$ $F(T)\}$.

Definition 2.3. A family $\left\{T_{i}: i \in \mathcal{N}\right\}$ of $N$-mappings on $C$, where $C$ be a nonempty subset of a separable Banach space $X$ with $\mathcal{F}=\cap_{i=1}^{N} F\left(T_{i}\right) \neq \emptyset$ is said to satisfy condition $(B)$ on $C$ if there is a nondecreasing function $f:[0, \infty) \rightarrow$ $[0, \infty)$ with $f(0)=0, f(r)>0$ for all $r \in(0, \infty)$ such that $a_{1}\left\|x-T_{1} x\right\|+$ $a_{2}\left\|x-T_{2} x\right\|+\cdots+a_{N}\left\|x-T_{N} x\right\| \geq f(d(x, \mathcal{F}))$ for all $x \in C$, where $d(x, \mathcal{F})=$ $\inf \{\|x-p\|: p \in \mathcal{F}\}$ and $a_{1}, a_{2}, \ldots, a_{N}$ are $N$ nonnegative real numbers such that $a_{1}+a_{2}+\cdots+a_{N}=1$.

Remark 2.4. condition (B) reduces to condition (A) when $T_{1}=T_{2}=\cdots=T_{N}=$ $T$.

In the sequel, we will need the following lemmas.

Lemma 2.5. (Tan and $X u[24]):$ Let $\left\{a_{n}\right\}_{n=1}^{\infty},\left\{\beta_{n}\right\}_{n=1}^{\infty}$ and $\left\{r_{n}\right\}_{n=1}^{\infty}$ be sequences of nonnegative real numbers satisfying

$$
a_{n+1} \leq\left(1+r_{n}\right) a_{n}+\beta_{n}, \forall n \in N .
$$

If $\sum_{n=1}^{\infty} r_{n}<\infty, \sum_{n=1}^{\infty} \beta_{n}<\infty$. Then

(i) $\lim _{n \rightarrow \infty} a_{n}$ exists. 
(ii) If $\liminf _{n \rightarrow \infty} a_{n}=0$, then $\lim _{n \rightarrow \infty} a_{n}=0$.

Lemma 2.6. (Schu [20]) Let $E$ be a uniformly convex Banach space and $0<$ $a \leq t_{n} \leq b<1$ for all $n \geq 1$. Suppose that $\left\{x_{n}\right\}$ and $\left\{y_{n}\right\}$ are sequences in $E$ satisfying

$$
\limsup _{n \rightarrow \infty}\left\|x_{n}\right\| \leq r, \quad \limsup _{n \rightarrow \infty}\left\|y_{n}\right\| \leq r, \quad \lim _{n \rightarrow \infty}\left\|t_{n} x_{n}+\left(1-t_{n}\right) y_{n}\right\|=r
$$

for some $r \geq 0$. Then

$$
\lim _{n \rightarrow \infty}\left\|x_{n}-y_{n}\right\|=0
$$

\section{Main Results}

In this section, we study an implicit random iteration scheme with errors to common random fixed point for a finite family of asymptotically quasi-nonexpansive random operators in uniformly convex Banach spaces. We also establish the necessary and sufficient condition for the convergence of this process to the common random fixed point of the above said finite family of random operators. Our results extend the corresponding results of Beg and Abbas [5] and Chang et al. [10].

Theorem 3.1. Let $X$ be a uniformly convex separable Banach space, and let $C$ be a nonempty closed and convex subset of $X$. Let $\left\{T_{i}: i \in \mathcal{N}\right\}$ be a finite family of asymptotically quasi-nonexpansive random operators from $\Omega \times C$ to $C$ with sequences of measurable mapping $r_{n_{i}}: \Omega \rightarrow[0, \infty)$ satisfying $\sum_{n=1}^{\infty} r_{n_{i}}(\omega)<\infty$ for each $\omega \in \Omega$ and for all $i \in \mathcal{N}$ and $\mathcal{F}=\cap_{i=1}^{N} R F\left(T_{i}\right) \neq \emptyset$. Let $\xi_{0}$ be a measurable mapping from $\Omega$ to $C$, then the sequence of random implicit iteration process with errors defined as by (2.5) converges to a common random fixed point of random operators $\left\{T_{i}: i \in \mathcal{N}\right\}$ in $C$ if and only if $\liminf _{n \rightarrow \infty} d\left(\xi_{n}(\omega), \mathcal{F}\right)=0$,

where $\left\{\beta_{n}\right\} \subset(s, 1-s)$ for some $s \in\left(0, \frac{1}{2}\right), \sum_{n=1}^{\infty} \gamma_{n}<\infty$ and $\left\{f_{n}(\omega)\right\}$ is arbitrary bounded sequence in $C$.

Proof. The necessity is obvious. Thus we will only prove the sufficiency. For any measurable mapping $\xi \in \mathcal{F}$, from $(2.5)$, where $n=(k-1) N+i, T_{n}=T_{n(\bmod N)}=$ $T_{i}, i \in \mathcal{N}$, it follows that 


$$
\begin{aligned}
\left\|\xi_{n}(\omega)-\xi(\omega)\right\|= & \left\|\alpha_{n} \xi_{n-1}(\omega)+\beta_{n} T_{i}^{k}\left(\omega, \xi_{n}(\omega)\right)+\gamma_{n} f_{n}(\omega)-\xi(\omega)\right\| \\
= & \| \alpha_{n}\left(\xi_{n-1}(\omega)-\xi(\omega)\right)+\beta_{n}\left(T_{i}^{k}\left(\omega, \xi_{n}(\omega)\right)-\xi(\omega)\right) \\
& +\gamma_{n}\left(f_{n}(\omega)-\xi(\omega)\right) \| \\
\leq & \alpha_{n}\left\|\xi_{n-1}(\omega)-\xi(\omega)\right\|+\beta_{n}\left\|T_{i}^{k}\left(\omega, \xi_{n}(\omega)\right)-\xi(\omega)\right\| \\
& +\gamma_{n}\left\|f_{n}(\omega)-\xi(\omega)\right\| \\
\leq & \alpha_{n}\left\|\xi_{n-1}(\omega)-\xi(\omega)\right\|+\beta_{n}\left(1+r_{k_{i}}(\omega)\right)\left\|\xi_{n}(\omega)-\xi(\omega)\right\| \\
& +\gamma_{n}\left\|f_{n}(\omega)-\xi(\omega)\right\| \\
\leq & \alpha_{n}\left\|\xi_{n-1}(\omega)-\xi(\omega)\right\|+\left(\beta_{n}+r_{k_{i}}(\omega)\right)\left\|\xi_{n}(\omega)-\xi(\omega)\right\| \\
& +\gamma_{n}\left\|f_{n}(\omega)-\xi(\omega)\right\| \\
\leq & \alpha_{n}\left\|\xi_{n-1}(\omega)-\xi(\omega)\right\|+\left(1-\alpha_{n}+r_{k_{i}}(\omega)\right)\left\|\xi_{n}(\omega)-\xi(\omega)\right\| \\
& +\gamma_{n}\left\|f_{n}(\omega)-\xi(\omega)\right\| .
\end{aligned}
$$

Since $\lim _{n \rightarrow \infty} \gamma_{n}=0$, there exists a natural number $n_{1}$ such that for $n>n_{1}, \gamma_{n} \leq \frac{s}{2}$. Hence

$$
\alpha_{n}=1-\beta_{n}-\gamma_{n} \geq 1-(1-s)-\frac{s}{2}=\frac{s}{2},
$$

for $n>n_{1}$. Thus, we have from (3.1) that

$$
\begin{aligned}
\alpha_{n}\left\|\xi_{n}(\omega)-\xi(\omega)\right\| \leq & \alpha_{n}\left\|\xi_{n-1}(\omega)-\xi(\omega)\right\|+r_{k_{i}}(\omega)\left\|\xi_{n}(\omega)-\xi(\omega)\right\| \\
& +\gamma_{n}\left\|f_{n}(\omega)-\xi(\omega)\right\|
\end{aligned}
$$

and

$$
\begin{aligned}
\left\|\xi_{n}(\omega)-\xi(\omega)\right\| \leq & \left\|\xi_{n-1}(\omega)-\xi(\omega)\right\|+\frac{r_{k_{i}}(\omega)}{\alpha_{n}}\left\|\xi_{n}(\omega)-\xi(\omega)\right\| \\
& +\frac{\gamma_{n}}{\alpha_{n}}\left\|f_{n}(\omega)-\xi(\omega)\right\| \\
\leq & \left\|\xi_{n-1}(\omega)-\xi(\omega)\right\|+\frac{2}{s} r_{k_{i}}(\omega)\left\|\xi_{n}(\omega)-\xi(\omega)\right\| \\
& +\frac{2}{s} \gamma_{n}\left\|f_{n}(\omega)-\xi(\omega)\right\| .
\end{aligned}
$$

Since $\sum_{n=1}^{\infty} r_{k_{i}}(\omega)<\infty$ for all $i \in \mathcal{N}, \lim _{n \rightarrow \infty} r_{n_{i}}(\omega)=0$ for each $i \in \mathcal{N}$. Hence there exists a natural number $n_{2}$, as $n>\frac{n_{2}}{N}+1$, that is, $n>n_{2}$ such that

$$
r_{n_{i}}(\omega) \leq \frac{s}{4}, \quad \forall i \in \mathcal{N}
$$

Then (3.2) becomes

$$
\left\|\xi_{n}(\omega)-\xi(\omega)\right\| \leq \frac{s}{s-2 r_{k_{i}}(\omega)}\left\|\xi_{n-1}(\omega)-\xi(\omega)\right\|+\frac{2 \gamma_{n}}{s-2 r_{k_{i}}(\omega)}\left\|f_{n}(\omega)-\xi(\omega)\right\| .
$$

Let 
CONVERGENCE OF IMPLICIT RANDOM...

$$
1+\theta_{k_{i}}(\omega)=\frac{s}{s-2 r_{k_{i}}(\omega)}=1+\frac{2 r_{k_{i}}(\omega)}{s-2 r_{k_{i}}(\omega)}
$$

Then

$$
\theta_{k_{i}}(\omega)=\frac{2 r_{k_{i}}(\omega)}{s-2 r_{k_{i}}(\omega)}<\frac{4}{s} r_{k_{i}}(\omega)
$$

Therefore

$$
\sum_{k=1}^{\infty} \theta_{k_{i}}(\omega)<\frac{4}{s} \sum_{k=1}^{\infty} r_{k_{i}}(\omega)<\infty, \quad \forall i \in \mathcal{N}
$$

and (3.3) becomes

$$
\begin{aligned}
\left\|\xi_{n}(\omega)-\xi(\omega)\right\| & \leq\left(1+\theta_{k_{i}}(\omega)\right)\left\|\xi_{n-1}(\omega)-\xi(\omega)\right\|+\frac{2 \gamma_{n}}{s-2 r_{k_{i}}(\omega)}\left\|f_{n}(\omega)-\xi(\omega)\right\| \\
& \leq\left(1+\theta_{k_{i}}(\omega)\right)\left\|\xi_{n-1}(\omega)-\xi(\omega)\right\|+\frac{4}{s} \gamma_{n} M
\end{aligned}
$$

where, $M=\sup _{n \geq 1}\left\|f_{n}(\omega)-\xi(\omega)\right\|$, since $\left\{f_{n}(\omega)\right\}$ is a bounded sequence in $C$. This implies that

$$
d\left(\xi_{n}(\omega), \mathcal{F}\right) \leq\left(1+\theta_{k_{i}}(\omega)\right) d\left(\xi_{n-1}(\omega), \mathcal{F}\right)+\frac{4}{s} \gamma_{n} M
$$

Since $\sum_{k=1}^{\infty} \theta_{k_{i}}(\omega)<\infty$ and $\sum_{k=1}^{\infty} \gamma_{n}<\infty$, it follows from Lemma 2.5, we know that $\lim _{n \rightarrow \infty} d\left(\xi_{n}(\omega), \mathcal{F}\right)=0$.

Next, we will prove that $\left\{\xi_{n}\right\}$ is a Cauchy sequence. Notice that when $x>0$, $1+x \leq e^{x}$, from (3.4) we have 


$$
\begin{aligned}
\left\|\xi_{n+m}(\omega)-\xi(\omega)\right\| \leq & \left(1+\theta_{k_{i}}(\omega)\right)\left\|\xi_{n+m-1}(\omega)-\xi(\omega)\right\|+\frac{4 M}{s} \gamma_{n+m} \\
\leq & \left(1+\theta_{k_{i}}(\omega)\right)\left[\left(1+\theta_{k_{i}}(\omega)\right)\left\|\xi_{n+m-2}(\omega)-\xi(\omega)\right\|\right. \\
& \left.+\frac{4 M}{s} \gamma_{n+m-1}\right]+\frac{4 M}{s} \gamma_{n+m} \\
\leq & \left(1+\theta_{k_{i}}(\omega)\right)^{2}\left[\left(1+\theta_{k_{i}}(\omega)\right)\left\|\xi_{n+m-3}(\omega)-\xi(\omega)\right\|\right. \\
& \left.+\frac{4 M}{s} \gamma_{n+m-2}\right]+\frac{4 M}{s}\left(1+\theta_{k_{i}}(\omega)\right)\left(\gamma_{n+m-1}+\gamma_{n+m}\right) \\
\leq & \left(1+\theta_{k_{i}}(\omega)\right)^{3}\left\|\xi_{n+m-3}(\omega)-\xi(\omega)\right\| \\
& +\frac{4 M}{s}\left(1+\theta_{k_{i}}(\omega)\right)^{3}\left(\gamma_{n+m-2}+\gamma_{n+m-1}+\gamma_{n+m}\right) \\
\leq & \ldots \\
\leq & \exp \left\{\sum_{i=1}^{N} \sum_{k=1}^{\infty} \theta_{k_{i}}(\omega)\right\}\left\|\xi_{n}(\omega)-\xi(\omega)\right\| \\
& +\frac{4 M}{s} \exp \left\{\sum_{i=1}^{N} \sum_{k=1}^{\infty} \theta_{k_{i}}(\omega)\right\} \sum_{j=n+1}^{n+m} \gamma_{j} \\
\leq & M^{\prime}\left\|\xi_{n}(\omega)-\xi(\omega)\right\|+\frac{4 M M^{\prime}}{s+\sum_{j=n+1}^{n+m}} \gamma_{j},
\end{aligned}
$$

for all $\xi \in \mathcal{F}$ and $m, n \in \mathbb{N}$, where $M^{\prime}=\exp \left\{\sum_{i=1}^{N} \sum_{k=1}^{\infty} \theta_{k_{i}}(\omega)\right\}<\infty$. Since $\lim _{n \rightarrow \infty} d\left(\xi_{n}(\omega), \mathcal{F}\right)=0$ and $\sum_{k=1}^{\infty} r_{k_{i}}(\omega)<\infty(i \in \mathcal{N})$, there exists a natural number $n_{1}$ such that for $n \geq n_{1}$,

$$
d\left(\xi_{n}(\omega), \mathcal{F}\right)<\frac{\varepsilon}{4 M^{\prime}} \text { and } \sum_{j=n_{1}+1}^{n+m} \gamma_{j} \leq \frac{s . \varepsilon}{16 M M^{\prime}}
$$

Thus there exists a point $\xi(\omega) \in \mathcal{F}$ such that $d\left(\xi_{n_{1}}(\omega), \xi(\omega)\right)<\frac{\varepsilon}{4 M^{\prime}}$ for each $\omega \in \Omega$. It follows from (3.5) that for all $n \geq n_{1}$ and $m \geq 1$, we have 


$$
\begin{aligned}
\left\|\xi_{n+m}(\omega)-\xi_{n}(\omega)\right\| \leq & \left\|\xi_{n+m}(\omega)-\xi(\omega)\right\|+\left\|\xi_{n}(\omega)-\xi(\omega)\right\| \\
\leq & M^{\prime}\left\|\xi_{n_{1}}(\omega)-\xi(\omega)\right\|+\frac{4 M M^{\prime}}{s} \sum_{j=n_{1}+1}^{n+m} \gamma_{j} \\
& +M^{\prime}\left\|\xi_{n_{1}}(\omega)-\xi(\omega)\right\|+\frac{4 M M^{\prime}}{s} \sum_{j=n_{1}+1}^{n+m} \gamma_{j} \\
< & M^{\prime} \cdot \frac{\varepsilon}{4 M^{\prime}}+\frac{4 M M^{\prime}}{s} \cdot \frac{s \cdot \varepsilon}{16 M M^{\prime}} \\
& +M^{\prime} \cdot \frac{\varepsilon}{4 M^{\prime}}+\frac{4 M M^{\prime}}{s} \cdot \frac{s \cdot \varepsilon}{16 M M^{\prime}} \\
= & \varepsilon .
\end{aligned}
$$

This implies that $\left\{\xi_{n}(\omega)\right\}$ is a Cauchy sequence for each $\omega \in \Omega$. Therefore, $\xi_{n}(\omega) \rightarrow p(\omega)$ for each $\omega \in \Omega$, where $p: \Omega \rightarrow \mathcal{F}$, being the limit of the measurable mappings, is also measurable. Now, $\lim _{n \rightarrow \infty} d\left(\xi_{n}(\omega), \mathcal{F}\right)=0$, for each $\omega \in \Omega$, and the set $\mathcal{F}$ is closed; we have $p \in \mathcal{F}$, that is, $p$ is a common random fixed point of $\left\{T_{i}: i \in \mathcal{N}\right\}$. This completes the proof.

Lemma 3.2. Let $X$ be a uniformly convex separable Banach space, and let $C$ be a nonempty closed and convex subset of $X$. Let $\left\{T_{i}: i \in \mathcal{N}\right\}$ be a finite family of uniformly L-Lipschitzian, asymptotically quasi-nonexpansive random operators from $\Omega \times C$ to $C$ with sequences of measurable mapping $r_{n_{i}}: \Omega \rightarrow[0, \infty)$ satisfying $\sum_{n=1}^{\infty} r_{n_{i}}(\omega)<\infty$ for each $\omega \in \Omega$ and for all $i \in \mathcal{N}$ and $\mathcal{F}=\cap_{i=1}^{N} R F\left(T_{i}\right) \neq \emptyset$. Let $\xi_{0}$ be a measurable mapping from $\Omega$ to $C$, and let the sequence of random implicit iteration process with errors defined as by (2.5). If $\liminf _{n \rightarrow \infty} d\left(\xi_{n}(\omega), \mathcal{F}\right)=0$, where $\left\{\beta_{n}\right\} \subset(s, 1-s)$ for some $s \in\left(0, \frac{1}{2}\right), \sum_{n=1}^{\infty} \gamma_{n}<\infty$ and $\left\{f_{n}(\omega)\right\}$ is arbitrary bounded sequence in $C$, then

$$
\lim _{n \rightarrow \infty}\left\|\xi_{n}(\omega)-T_{n}\left(\omega, \xi_{n}(\omega)\right)\right\|=0
$$

for each $\omega \in \Omega$.

Proof. It follows from (3.4), and Lemma 2.5, that $\lim _{n \rightarrow \infty}\left\|\xi_{n}(\omega)-\xi(\omega)\right\|$ exists for any $\xi \in \mathcal{F}$. Since $\left\{\xi_{n}(\omega)-\xi(\omega)\right\}$ is a convergent sequence, without loss of generality, we can assume that

$$
\lim _{n \rightarrow \infty}\left\|\xi_{n}(\omega)-\xi(\omega)\right\|=d_{\omega}
$$

where $d_{\omega} \geq 0$. Observe that

$$
\begin{aligned}
\left\|\xi_{n}(\omega)-\xi(\omega)\right\|= & \left\|\alpha_{n} \xi_{n-1}(\omega)+\beta_{n} T_{i}^{k}\left(\omega, \xi_{n}(\omega)\right)+\gamma_{n} f_{n}(\omega)-\xi(\omega)\right\| \\
= & \| \beta_{n}\left[T_{i}^{k}\left(\omega, \xi_{n}(\omega)\right)-\xi(\omega)+\gamma_{n}\left(f_{n}(\omega)-\xi_{n-1}(\omega)\right)\right] \\
& +\left(1-\beta_{n}\right)\left[\xi_{n-1}(\omega)-\xi(\omega)+\gamma_{n}\left(f_{n}(\omega)-\xi_{n-1}(\omega)\right)\right] \| .
\end{aligned}
$$


From $\sum_{n=1}^{\infty} \gamma_{n}<\infty$ and (3.7), it follows that

$$
\begin{aligned}
\limsup _{n \rightarrow \infty} \| \xi_{n-1}(\omega)-\xi(\omega)+ & \gamma_{n}\left(f_{n}(\omega)-\xi_{n-1}(\omega)\right) \| \\
\leq & \limsup _{n \rightarrow \infty}\left[\left\|\xi_{n-1}(\omega)-\xi(\omega)\right\|\right. \\
& \left.+\gamma_{n}\left\|f_{n}(\omega)-\xi_{n-1}(\omega)\right\|\right] \\
\leq & d_{\omega},
\end{aligned}
$$

and hence

$$
\begin{aligned}
\limsup _{n \rightarrow \infty} \| T_{n}^{k}\left(\omega, \xi_{n}(\omega)\right)-\xi(\omega)+ & \gamma_{n}\left(f_{n}(\omega)-\xi_{n-1}(\omega)\right) \| \\
\leq & \limsup _{n \rightarrow \infty}\left[\left\|T_{n}^{k}\left(\omega, \xi_{n}(\omega)\right)-\xi(\omega)\right\|\right. \\
& \left.+\gamma_{n}\left\|f_{n}(\omega)-\xi_{n-1}(\omega)\right\|\right] \\
\leq & \limsup _{n \rightarrow \infty}\left[\left(1+r_{k_{n}}(\omega)\right) \| \xi_{n}(\omega)\right)-\xi(\omega) \| \\
& \left.+\gamma_{n}\left\|f_{n}(\omega)-\xi_{n-1}(\omega)\right\|\right] \\
\leq & d_{\omega},
\end{aligned}
$$

where $n=(k-1) N+i$.

Therefore from (3.7) - (3.10) and Lemma 2.6, we have that

$$
\lim _{n \rightarrow \infty}\left\|T_{n}^{k}\left(\omega, \xi_{n}(\omega)\right)-\xi_{n-1}(\omega)\right\|=0
$$

for each $\omega \in \Omega$.

Moreover, since

$$
\begin{aligned}
\left\|\xi_{n}(\omega)-\xi_{n-1}(\omega)\right\|= & \left\|\alpha_{n} \xi_{n-1}(\omega)+\beta_{n} T_{n}^{k}\left(\omega, \xi_{n}(\omega)\right)+\gamma_{n} f_{n}(\omega)-\xi_{n-1}(\omega)\right\| \\
= & \left\|\beta_{n}\left[T_{n}^{k}\left(\omega, \xi_{n}(\omega)\right)-\xi_{n-1}(\omega)\right]+\gamma_{n}\left[f_{n}(\omega)-\xi_{n-1}(\omega)\right]\right\| \\
\leq & \beta_{n}\left\|T_{n}^{k}\left(\omega, \xi_{n}(\omega)\right)-\xi_{n-1}(\omega)\right\| \\
& +\gamma_{n}\left\|f_{n}(\omega)-\xi_{n-1}(\omega)\right\|
\end{aligned}
$$

hence by (3.11), we obtain

$$
\lim _{n \rightarrow \infty}\left\|\xi_{n}(\omega)-\xi_{n-1}(\omega)\right\|=0,
$$

for each $\omega \in \Omega$ and $\left\|\xi_{n}(\omega)-\xi_{n+l}(\omega)\right\| \rightarrow 0$, for each $\omega \in \Omega$ and $l<2 N$. Now, for $n>N$, we have 


$$
\begin{aligned}
\left\|\xi_{n-1}(\omega)-T_{n}\left(\omega, \xi_{n}(\omega)\right)\right\| \leq & \left\|\xi_{n-1}(\omega)-T_{n}^{k}\left(\omega, \xi_{n}(\omega)\right)\right\| \\
& +\left\|T_{n}^{k}\left(\omega, \xi_{n}(\omega)\right)-T_{n}\left(\omega, \xi_{n}(\omega)\right)\right\| \\
\leq & \left\|\xi_{n-1}(\omega)-T_{n}^{k}\left(\omega, \xi_{n}(\omega)\right)\right\| \\
& +L\left\|T_{n}^{k-1}\left(\omega, \xi_{n}(\omega)\right)-\xi_{n}(\omega)\right\| \\
\leq & \left\|\xi_{n-1}(\omega)-T_{n}^{k}\left(\omega, \xi_{n}(\omega)\right)\right\| \\
& +L\left\|T_{n}^{k-1}\left(\omega, \xi_{n}(\omega)\right)-T_{n-N}^{k-1}\left(\omega, \xi_{n-N}(\omega)\right)\right\| \\
& +L\left[\left\|T_{n-N}^{k-1}\left(\omega, \xi_{n-N}(\omega)\right)-\xi_{(n-N)-1}(\omega)\right\|\right. \\
& \left.+\left\|\xi_{(n-N)-1}(\omega)-\xi_{n}(\omega)\right\|\right] .
\end{aligned}
$$

Since for each $n>N, n \equiv(n-N) \bmod N$. Thus $T_{n}=T_{n-N}$, therefore

$$
\begin{aligned}
\left\|\xi_{n-1}(\omega)-T_{n}\left(\omega, \xi_{n}(\omega)\right)\right\| \leq & \left\|\xi_{n-1}(\omega)-T_{n}^{k}\left(\omega, \xi_{n}(\omega)\right)\right\|+L^{2}\left\|\xi_{n}(\omega)-\xi_{n-N}(\omega)\right\| \\
& +L\left\|T_{n-N}^{k-1}\left(\omega, \xi_{n-N}(\omega)\right)-\xi_{(n-N)-1}(\omega)\right\| \\
& +L\left\|\xi_{(n-N)-1}(\omega)-\xi_{n}(\omega)\right\| .
\end{aligned}
$$

This implies that

$$
\lim _{n \rightarrow \infty}\left\|\xi_{n-1}(\omega)-T_{n}\left(\omega, \xi_{n}(\omega)\right)\right\|=0
$$

for each $\omega \in \Omega$. Now

$$
\begin{aligned}
\left\|\xi_{n}(\omega)-T_{n}\left(\omega, \xi_{n}(\omega)\right)\right\| \leq & \left\|\xi_{n-1}(\omega)-\xi_{n}(\omega)\right\| \\
& +\left\|\xi_{n-1}(\omega)-T_{n}\left(\omega, \xi_{n}(\omega)\right)\right\| .
\end{aligned}
$$

Hence

$$
\lim _{n \rightarrow \infty}\left\|\xi_{n}(\omega)-T_{n}\left(\omega, \xi_{n}(\omega)\right)\right\|=0
$$

for each $\omega \in \Omega$.

Theorem 3.3. Let $X$ be a uniformly convex separable Banach space, and let $C$ be a nonempty closed and convex subset of $X$. Let $\left\{T_{i}: i \in \mathcal{N}\right\}$ be a finite family of uniformly L-Lipschitzian, asymptotically quasi-nonexpansive random operators from $\Omega \times C$ to $C$ with sequences of measurable mapping $r_{n_{i}}: \Omega \rightarrow[0, \infty)$ satisfying $\sum_{n=1}^{\infty} r_{n_{i}}(\omega)<\infty$ for each $\omega \in \Omega$ and for all $i \in \mathcal{N}$ and $\mathcal{F}=\cap_{i=1}^{N} R F\left(T_{i}\right) \neq \emptyset$. Suppose there is one member $T$ in the family $\left\{T_{i}: i \in \mathcal{N}\right\}$ which is semi-compact random operator. Let $\xi_{0}$ be a measurable mapping from $\Omega$ to $C$. Then the sequence of random implicit iteration process with errors defined as by (2.5) converges to a common random fixed point of random operators $\left\{T_{i}: i \in \mathcal{N}\right\}$, where $\left\{\beta_{n}\right\} \subset$ $(s, 1-s)$ for some $s \in\left(0, \frac{1}{2}\right), \sum_{n=1}^{\infty} \gamma_{n}<\infty$ and $\left\{f_{n}(\omega)\right\}$ is arbitrary bounded sequence in $C$. 
Proof. For any given $\xi(\omega) \in \mathcal{F}$, we note that

$$
\lim _{n \rightarrow \infty}\left\|\xi_{n}(\omega)-\xi(\omega)\right\|=d_{\omega}
$$

where $d_{\omega} \geq 0$. By Lemma 3.2, we know that

$$
\lim _{n \rightarrow \infty}\left\|\xi_{n}(\omega)-T_{n}\left(\omega, \xi_{n}(\omega)\right)\right\|=0,
$$

for each $\omega \in \Omega$. Consequently, for any $j \in \mathcal{N}$,

$$
\begin{aligned}
\left\|\xi_{n}(\omega)-T_{n+j}\left(\omega, \xi_{n}(\omega)\right)\right\| \leq & \left\|\xi_{n}(\omega)-\xi_{n+j}(\omega)\right\| \\
& +\left\|\xi_{n+j}(\omega)-T_{n+j}\left(\omega, \xi_{n+j}(\omega)\right)\right\| \\
& +\left\|T_{n+j}\left(\omega, \xi_{n+j}(\omega)\right)-T_{n+j}\left(\omega, \xi_{n}(\omega)\right)\right\| \\
\leq & (1+L)\left\|\xi_{n}(\omega)-\xi_{n+j}(\omega)\right\| \\
& +\left\|\xi_{n+j}(\omega)-T_{n+j}\left(\omega, \xi_{n+j}(\omega)\right)\right\| \\
& \rightarrow 0,
\end{aligned}
$$

as $n \rightarrow \infty$ for each $\omega \in \Omega$ and $j \in \mathcal{N}$, where $\mathcal{N}=\{1,2, \ldots, N\}$.

Consequently, $\left\|\xi_{n}(\omega)-T_{j}\left(\omega, \xi_{n}(\omega)\right)\right\| \rightarrow 0$ as $n \rightarrow \infty$ for each $\omega \in \Omega$ and $j \in \mathcal{N}$. Assume that $T_{j}$ is a semi-compact random operator. Therefore, there exists a subsequence $\left\{\xi_{n_{k}}\right\}$ of $\left\{\xi_{n}\right\}$ and a measurable mapping $\xi_{0}: \Omega \rightarrow C$ such that $\xi_{n_{k}}$ converges pointwise to $\xi_{0}$. Now

$$
\lim _{n \rightarrow \infty}\left\|\xi_{n_{k}}(\omega)-T_{j}\left(\omega, \xi_{n_{k}}(\omega)\right)\right\|=\left\|\xi_{0}(\omega)-T_{j}\left(\omega, \xi_{0}(\omega)\right)\right\|=0
$$

for each $\omega \in \Omega$, and $j \in \mathcal{N}$. It implies that $\xi_{0} \in \mathcal{F}$, and so $\liminf _{n \rightarrow \infty} d\left(\xi_{n}(\omega), \mathcal{F}\right)=0$. Hence, by Theorem 3.1, we obtain that $\left\{\xi_{n}\right\}$ converges to a point in $\mathcal{F}$. This completes the proof.

Theorem 3.4. Let $X$ be a uniformly convex separable Banach space, and let $C$ be a nonempty closed and convex subset of $X$. Let $\left\{T_{i}: i \in \mathcal{N}\right\}$ be a finite family of uniformly L-Lipschitzian, asymptotically quasi-nonexpansive random operators from $\Omega \times C$ to $C$ with sequences of measurable mapping $r_{n_{i}}: \Omega \rightarrow[0, \infty)$ satisfying $\sum_{n=1}^{\infty} r_{n_{i}}(\omega)<\infty$ for each $\omega \in \Omega$ and for all $i \in \mathcal{N}$ and $\mathcal{F}=\cap_{i=1}^{N} R F\left(T_{i}\right) \neq \emptyset$. Suppose the family $\left\{T_{i}: i \in \mathcal{N}\right\}$ satisfies the condition $(B)$. Let $\xi_{0}$ be a measurable mapping from $\Omega$ to $C$. Then the sequence of random implicit iteration process with errors defined as by (2.5) converges to a common random fixed point of random operators $\left\{T_{i}: i \in \mathcal{N}\right\}$, where $\left\{\beta_{n}\right\} \subset(s, 1-s)$ for some $s \in\left(0, \frac{1}{2}\right), \sum_{n=1}^{\infty} \gamma_{n}<\infty$ and $\left\{f_{n}(\omega)\right\}$ is arbitrary bounded sequence in $C$.

Proof. Let $\xi(\omega) \in \mathcal{F}$. Then it follows from (3.19) and the condition $(B)$ that

$$
\begin{aligned}
d\left(\xi_{n}(\omega), \mathcal{F}\right) \leq & a_{1}\left\|\xi_{n}(\omega)-T_{1}\left(\omega, \xi_{n}(\omega)\right)\right\|+a_{2}\left\|\xi_{n}(\omega)-T_{2}\left(\omega, \xi_{n}(\omega)\right)\right\| \\
& +\cdots+a_{N}\left\|\xi_{n}(\omega)-T_{N}\left(\omega, \xi_{n}(\omega)\right)\right\|
\end{aligned}
$$


for each $n \geq 1$, and $\omega \in \Omega$. Since $\left\|\xi_{n}(\omega)-T_{j}\left(\omega, \xi_{n}(\omega)\right)\right\| \rightarrow 0$ as $n \rightarrow \infty$, for each $\omega \in \Omega$ and $j \in \mathcal{N}$, we have

$$
\lim _{n \rightarrow \infty} f\left(d\left(\xi_{n}(\omega), \mathcal{F}\right)\right)=0 .
$$

Since $f$ is nondecreasing on $[0, \infty)$ with $f(0)=0$ and $f(r)>0$, for all $r \in(0, \infty)$, it follows that

$$
\lim _{n \rightarrow \infty} d\left(\xi_{n}(\omega), \mathcal{F}\right)=0 .
$$

Let $\varepsilon>0$. Since $\lim _{n \rightarrow \infty} d\left(\xi_{n}(\omega), \mathcal{F}\right)=0$, for each $\omega \in \Omega$, there exists a natural number $n_{1}$ such that for $n \geq n_{1}, d\left(\xi_{n}(\omega), \mathcal{F}\right)<\frac{\varepsilon}{3}$, for each $\omega \in \Omega$. In particular, there exists a point $\xi^{*}(\omega) \in \mathcal{F}$ such that $\left\|\xi_{n_{1}}(\omega)-\xi^{*}(\omega)\right\|<\frac{\varepsilon}{2}$. Now for $n \geq n_{1}$ and for all $m \geq 1$, we have

$$
\begin{aligned}
\left\|\xi_{n+m}(\omega)-\xi_{n}(\omega)\right\| & \leq\left\|\xi_{n+m}(\omega)-\xi^{*}(\omega)\right\|+\left\|\xi_{n}(\omega)-\xi^{*}(\omega)\right\| \\
& \leq\left\|\xi_{n_{1}}(\omega)-\xi^{*}(\omega)\right\|+\left\|\xi_{n_{1}}(\omega)-\xi^{*}(\omega)\right\| \\
& <\frac{\varepsilon}{2}+\frac{\varepsilon}{2}=\varepsilon
\end{aligned}
$$

This implies that $\left\{\xi_{n}(\omega)\right\}$ is a Cauchy sequence for each $\omega \in \Omega$. By the completeness of the space $X$, there exists a measurable mapping $p: \Omega \rightarrow C$ such that $\lim _{n \rightarrow \infty} \xi_{n}(\omega)=p(\omega)$, for all $\omega \in \Omega$. Next, we prove that $p(\omega) \in \mathcal{F}$. Let $\varepsilon_{1}>0$ be given. Then there exists a natural number $n_{2}$ such that $\left\|\xi_{n}(\omega)-p(\omega)\right\|<\frac{\varepsilon_{1}}{4}$ for all $n \geq n_{2}$. Since $\lim _{n \rightarrow \infty} d\left(\xi_{n}(\omega), \mathcal{F}\right)=0$, there exists a natural number $n_{3} \geq n_{2}$ such that for all $n \geq n_{3}$ we have $d\left(\xi_{n}(\omega), \mathcal{F}\right)<\frac{\varepsilon_{1}}{4}$ and in particular we have $d\left(\xi_{n_{3}}(\omega), \mathcal{F}\right)<\frac{\varepsilon_{1}}{4}$. This implies that there exists $q(\omega) \in \mathcal{F}$ such that $\left\|\xi_{n_{3}}(\omega)-q(\omega)\right\|<\frac{\varepsilon_{1}}{4}$. Then for each $i \in \mathcal{N}$ and $n \geq n_{3}$, we have

$$
\begin{aligned}
\left\|T_{i}(\omega, p(\omega))-p(\omega)\right\| & \leq\left\|T_{i}(\omega, p(\omega))-q(\omega)\right\|+\|q(\omega)-p(\omega)\| \\
& \leq 2\|p(\omega)-q(\omega)\| \\
& \leq 2\left[\left\|p(\omega)-\xi_{n_{3}}(\omega)\right\|+\left\|\xi_{n_{3}}(\omega)-q(\omega)\right\|\right] \\
& <2\left(\frac{\varepsilon_{1}}{4}+\frac{\varepsilon_{1}}{4}\right)=\varepsilon_{1} .
\end{aligned}
$$

Therefore, $T_{i}(\omega, p(\omega))=p(\omega)$, for all $i \in\{1,2, \ldots, N\}=\mathcal{N}$, that is, $p(\omega)$ is a common random fixed point of the random operators $\left\{T_{i}: i \in \mathcal{N}\right\}$. This completes the proof.

Remark 3.5. Our results extend and improve the corresponding results of Beg and Abbas [5] to the case of implicit random iteration process with errors for a finite family of asymptotically quasi-nonexpansive random operators.

Remark 3.6. Theorem 3.1 is a stochastic version of Theorem 2.1 of Kim et al. [16]. 


\section{REFERENCES}

1. V.H. Badshah and F. Sayyed, Common random fixed points of random multivalued operators on polish spaces, Indian J. Pure and Appl. Maths. 33(4) (2002), 573-582. 1

2. I. Beg, Random fixed points of random operators satisfying semicontractivity conditions, Maths. Japonica 46(1) (1997), 151-155. 1

3. I. Beg, Approximation of random fixed points in normed spaces, Nonlinear Anal. 51(8) (2002), 1363-1372. 1

4. I. Beg and M. Abbas, Equivalence and stability of random fixed point iterative procedures, J. Appl. Maths. Stoch. Anal. (2006), Article ID 23297, 19 pages. 1

5. I. Beg and M. Abbas, Iterative procedures for solutions of random operator equations in Banach spaces, J. Math. Anal. Appl. 315(1) (2006), 181-201. 1, 1, 3, 3.5

6. I. Beg and M. Abbas, Convergence of iterative algorithms to common random fixed points of random operators, J. Appl. Maths. Stoch. Anal. (2006), Article ID 89213, pages 1-16. 1

7. I. Beg and N. Shahzad, Common random fixed points of random multivalued operators on metric spaces, Boll. della Unione Math. Italiana 9(3) (1995), 493-503. 1

8. A.T. Bharucha-Reid, Random Integral equations, Mathematics in Science and Engineering, vol. 96, Academic Press, New York, 1972. 1

9. A.T. Bharucha-Reid, Fixed point theorems in Probabilistic analysis, Bull. Amer. Math. Soc. 82(5) (1976), 641-657. 1

10. S.S. Chang; K.K. Tan; H.W.J. Lee and C.K. Chan, On the convergence of implicit iteration process with error for a finite family of asymptotically nonexpansive mappings, J. Maths. Anal. Appl. 313(1) (2006), 273-283. 3

11. B.S. Choudhury, Convergence of a random iteration scheme to a random fixed point, J. Appl. Maths. Stoch. Anal. 8(2) (1995), 139-142. 1

12. O. Hanš, Reduzierende zufallige transformationen, Czech. Math. J. 7(82) (1957), 154-158. 1

13. O. Hanš, Random operator equations, Proc. of the 4th Berkeley Sympos. on Mathematical Statistics and Probability, Vol. II, University of California Press, California, 1961, pp. 185202. 1

14. C.J. Himmelberg, Measurable relations, Fundamenta Mathematicae 87 (1975), 53-72. 1

15. S. Itoh, Random fixed point theorems with an application to random differential equations in Banach spaces, J. Math. Anal. Appl. 67(2) (1979), 261-273. 1

16. J.K. Kim; K.S. Kim and S.M. Kim, Convergence theorems of implicit iteration process for a finite family of asymptotically quasi-nonexpansive mappings in convex metric spaces, Nonlinear Anal. and Convex Anal. RIMS Vol. 1484 (2006), 40-51. 3.6

17. N.S. Papageorgiou, Random fixed point theorems for measurable multifunctions in Banach spaces, Proc. Amer. Math. Soc. 97(3) (1986), 507-514. 1

18. N.S. Papageorgiou, On measurable multifunctions with stochastic domain, J. Austra. Math. Soc. Ser. A 45(2) (1988), 204-216. 1

19. R. Penaloza, A characterization of renegotiation proof contracts via random fixed points in Banach spaces, working paper 269, Department of Economics, Univ. Brasilia, Brasilia, December 2002. 1

20. J. Schu, Weak and strong convergence theorems to fixed points of asymptotically nonexpansive mappings, Bull. Austral. Math. Soc. 43 (1991), 153-159. 2.6

21. V.M. Sehgal and S.P. Singh, On random approximations and a random fixed point theorem for set valued mappings, Proc. Amer. Math. Soc. 95(1) (1985), 91-94. 1

22. H.F. Senter and W.G. Dotson, Approximating fixed points of nonexpansive mappings, Proc. Amer. Math. Soc. 44 (1974), 375-380. 2

23. Z.H. Sun, Strong convergence of an implicit iteration process for a finite family of asymptotically quasi-nonexpansive mappings, J. Math. Anal. Appl. 286 (2003), 351-358. 1, 1, 2 
24. K.K. Tan and H.K. Xu, Approximating fixed points of nonexpansive mappings by the Ishikawa iteration process, J. Math. Anal. Appl. 178 (1993), 301-308. 2.5

25. D.H. Wagner, Survey of measurable selection theorem, SIAM J. Contr. Optim. 15(5) (1977), 859-903. 1

26. R. Wittmann, Approximation of fixed points of nonexpansive mappings, Arch. Math. $\mathbf{5 8}$ (1992), 486-491. 1

27. H.K. Xu and R.G. Ori, An implicit iteration process for nonexpansive mappings, Numer. Funct. Anal. Optim. 22 (2001), 767-773. 1, 1,2

Department of Mathematics and Information Technology, Govt. Nagarjuna P.G. College of Science, RAipur (C.G.), India.

E-mail address: saluja_1963@rediffmail.com 